Editorials

\title{
The Rough Intuitionistic Fuzzy Zweier Lacunary Ideal Convergence of Triple Sequence Spaces
}

\author{
${ }^{1}$ Ayhan Esi, ${ }^{2}$ Nagarajan Subramanian and ${ }^{3}$ Vakeel Ahmad Khan \\ ${ }^{I}$ Department of Mathematics, Adiyaman University, 02040, Adiyaman, Turkey \\ ${ }^{2}$ Department of Mathematics, SASTRA Deemed University, Thanjavur. 613401, India \\ ${ }^{3}$ Department of Mathematics, Aligarh Muslim University, Aligarh, India
}

\author{
Article history \\ Received: 24-04-2018 \\ Revised: $14-05-2018$ \\ Accepted: 30-05-2018 \\ Corresponding Author: \\ Ayhan Esi \\ Department of Mathematics, \\ Adiyaman University, 02040, \\ Adiyaman, Turkey \\ Email: aesi23@hotmail.com
}

\begin{abstract}
We introduced and studied the concept of $I$-convergence of triple sequences in metric spaces where $I$ is an ideal. The concept of $I$ convergence has a wide application in the field of number theory, trigonometric series, summability theory, probability theory, optimization and approximation theory. In this article, we introduce rough intuitionistic fuzzy Lacunary ideal convergent of triple sequence spaces via zwier operators. We discuss general topological properties.
\end{abstract}

Keywords: Rough Intuitionistic Fuzzy Metric Space, I-convergence, $I_{\theta}$ Cauchy, Zweier Triple Sequence Spaces

\section{Introduction}

The idea of rough convergence was first introduced by Phu $(2001 ; 2002 ; 2003)$ in finite dimensional normed spaces. He showed that the set LIM $_{x}^{r}$ is bounded, closed and convex; and he introduced the notion of rough Cauchy sequence. He also investigated the relations between rough convergence and other convergence types and the dependence of $\operatorname{LIM}_{x}^{r}$ on the roughness of degree $r$.

Aytar (2008a) studied of rough statistical convergence and defined the set of rough statistical limit points of a sequence and obtained two statistical convergence criteria associated with this set and prove that this set is closed and convex. Also, Aytar (2008b) studied that the $r$-limit set of the sequence is equal to intersection of these sets and that $r$-core of the sequence is equal to the union of these sets. Dündar and Cakan (2014) investigated of rough ideal convergence and defined the set of rough ideal limit points of a sequence The notion of $I$-convergence of a triple sequence spaces which is based on the structure of the ideal $I$ of subsets of $\mathbb{N} \times \mathbb{N} \times \mathbb{N}$, where $\mathbb{N}$ is the set of all natural numbers, is a natural generalization of the notion of convergence and statistical convergence.

Let $K$ be a subset of the set of positive integers $\mathbb{N} \times \mathbb{N} \times \mathbb{N}$ and let us denote the set $K_{i k \ell}=\{(m, n, k) \in$ $K: m \leq i, n \leq j, k \leq \ell$. Then the natural density of $K$ is given by:

$$
\delta(K)=\lim _{i, j, \ell \rightarrow \infty} \frac{\left|K_{i j \ell}\right|}{i j \ell},
$$

where, $\left|K_{i j \ell}\right|$ denotes the number of elements in $K_{i j \ell}$.

A triple sequence (real or complex) can be defined as a function $\mathrm{x}: \mathbb{N} \times \mathbb{N} \times \mathbb{N} \rightarrow \mathbb{R}(\mathbb{C})$, where $\mathbb{N}, \mathbb{R}$ and $\mathbb{C}$ denote the set of natural numbers, real numbers and complex numbers respectively. The different types of notions of triple sequence was introduced and investigated at the initial by Sahiner et al. (2007; Sahiner and Tripathy, 2008) Esi (2014; Esi and Necdet Catalbas, 2014; Esi and Savas, 2015; Esi et al., 2016; 2018), Dutta et al. (2013), Subramanian and Esi (2015), Debnath et al. (2015) and many others.

Let $\left(x_{m n k}\right)$ be a triple sequence of real or complex numbers. Then the series $\sum_{m, n, k=1}^{\infty} x_{m n k}$ is called a triple series. The triple series $\sum_{m, n, k=1}^{\infty} x_{m n k}$ give one space is said to be convergent if and only if the triple sequence $\left(S_{m n k}\right)$ is convergent, where:

$$
S_{m n k}=\sum_{i, j, q=1}^{m, n, k} x_{i j q}(m, n, k=1,2,3 \ldots) .
$$

A sequence $x=\left(x_{m n k}\right)$ is said to be triple analytic if:

$$
\sup _{m, n, k}\left|x_{m n k}\right|^{\frac{1}{m+n+k}}<\infty .
$$


The vector space of all triple analytic sequences are usually denoted by $\Lambda^{3}$. A sequence $x=\left(x_{m n k}\right)$ is called triple entire sequence if:

$$
\left|x_{m n k}\right|^{\frac{1}{m+n+k}} \rightarrow 0 \text { as } m, n, k \rightarrow \infty
$$

The vector space of all triple entire sequences are usually denoted by $\Gamma^{3}$. Let the set of sequences with this property be denoted by $\Lambda^{3}$ and $\Gamma^{3}$ is a metric space with the metric:

$$
d(x, y)=\sup _{m, n, k}\left\{\left|x_{m n k}-y_{m n k}\right|^{\frac{1}{m+n+k}}: m, n, k: 1,2,3, \ldots\right\},
$$

for all $x=\left\{x_{m n k}\right\}$ and $y=\left\{y_{m n k}\right\}$ in $\Gamma^{3}$. Let $\phi=\{$ finite sequences $\}$.

Let $\lambda$ and $\mu$ be two sequence spaces and $A=\left(a_{k \ell}^{m n}\right)$ is an infinite four dimensional matrix of real or complex numbers $a_{k \ell}^{m n}$, where $k, \ell, m, n \in \mathbb{N}$. Then we say that $A$ defines a matrix mapping from $\lambda$ to $\mu$ and we do not it by writing $A: \lambda \rightarrow \mu$.

If for every sequence $x=\left(x_{m n k}\right) \in \lambda$ the sequence $A x=$ $\left\{A(A x)_{m n}\right\} \in \mu$, then $A$ transform of $x \in \mu$, where $(A x)_{m n k}=$ $\sum_{m} \sum_{n} \sum_{k} a_{k \ell}^{m n} x_{m n k},(k, \ell \in \mathbb{N})$.

By $(\lambda: \mu)$, we denote the class of matrices $A$ such that $A: \lambda \rightarrow \mu$. Thus $a \in(\lambda, \mu)$ if and only if $(A x) \in \mu$ for every $x \in \lambda$.

The approach of constructing the new sequence spaces by means of the matrix domain of a particular limitation method.

The $Z^{p}$-transformation of the sequence $x=\left(x_{m n k}\right)$, (i.e.,) $y_{i j k}=p x_{i j k}+(1-p) x_{i-1 j-1 k-1} . Z^{p}$ denotes the matrix $Z^{p}$ $=\left(Z_{m n k}^{i j k}\right)$ defined by:

$$
Z_{m n k}^{i j k}= \begin{cases}p, & \text { if }(m n k-i j k) \\ 1-p, & \text { if }(i-1=m, j-1=n, k-1=k) \\ 0, \text { otherwise } & \end{cases}
$$

The Zweier sequence space $Z_{\Gamma^{3}}$ as follows:

$$
Z_{\Gamma^{3}}=\left\{x=\left(x_{m n k}\right) \in \omega^{3}: Z^{p} x \in \Gamma^{3}\right\}
$$

In this study, we are working on to connect to studies which was mentioned above in order to introduce rough intuitionistic triple sequence spaces and study some algebraic and topological properties on these spaces.

A fuzzy number $X$ is a fuzzy subset of the real $\mathbb{R}^{3}$; which is normal fuzzy convex, upper semi-continuous and the $X^{0}$ is bounded where $X^{0} ;=\operatorname{cl}\left\{x \in \mathbb{R}^{3}: X(x)>0\right\}$ and $c l$ is the closure operator. These properties imply that for each $\alpha \in(0,1]$, the $\alpha$-level set $X^{\alpha}$ defined by:

$$
X^{\alpha}=\left\{x \in \mathbb{R}^{3}: X(x) \geq \alpha\right\}=\left[\underline{X}^{\alpha}, \bar{X}^{\alpha}\right]
$$

is a non empty compact convex subset of $\mathbb{R}^{3}$.

The supremum metric $d$ on the set $L\left(\mathbb{R}^{3}\right)$ is defined by:

$$
d(X, Y)=\sup _{\alpha \in[0,1]} \max \left(\left|\underline{X}^{\alpha}-\underline{Y}^{\alpha}\right|,\left|\bar{X}^{\alpha}-\bar{Y}^{\alpha}\right|\right)
$$

Now, given $X, Y \in L\left(\mathbb{R}^{3}\right)$, we define $X \leq Y$ if $\underline{X}^{\alpha} \leq \underline{Y}^{\alpha}$ and $\bar{X}^{\alpha} \leq \bar{Y}^{\alpha}$ for each $\alpha \in[0$,$] .$

We write $X \leq Y$ if $X \leq Y$ and there exists an $\alpha_{0} \in[0,1]$ such that $\underline{X}^{\alpha_{0}} \leq \underline{Y}^{\alpha_{0}}$ or $\bar{X}^{\alpha_{0}} \leq \bar{Y}^{\alpha_{0}}$.

A subset $E$ of $L\left(\mathbb{R}^{3}\right)$ is said to be bounded above if there exists a fuzzy number $\mu$, called an upper bound of $E$, such that $X \leq \mu$ for every $X \in E$. $\mu$ is called the least upper bound of $E$ if $\mu$ is an upper bound and $\mu \leq \mu^{\prime}$ for all upper bounds $\mu^{\prime}$.

A lower bound and the greatest lower bound are defined similarly. $E$ is said to be bounded if it is both bounded above and below.

The notions of least upper bound and the greatest lower bound have been defined only for bounded sets of fuzzy numbers. If the set $E \subset L\left(\mathbb{R}^{3}\right)$ is bounded then its supremum and infimum exist.

The limit infimum and limit supremum of a triple sequence spaces $\left(X_{m n k}\right)$ is defined by:

$$
\begin{aligned}
& \lim _{m n k \rightarrow \infty} \inf X_{m n k}:=\inf A_{X} . \\
& \lim _{m n k \rightarrow \infty} \sup X_{m n k}:=\sup B_{X} .
\end{aligned}
$$

Where:

$$
\begin{aligned}
& A_{X}:=\left\{\mu \in L\left(\mathbb{R}^{3}\right): \text { The set }\left\{(m, n, k) \in \mathbb{N}^{3}: X_{m n k}<\mu\right\} \text { is infinite }\right\} \\
& B_{X}:=\left\{\mu \in L\left(\mathbb{R}^{3}\right): \text { The set }\left\{(m, n, k) \in \mathbb{N}^{3}: X_{m n k}<\mu\right\} \text { is infinite }\right\}
\end{aligned}
$$

Now, given two fuzzy numbers $X, Y \in L\left(\mathbb{R}^{3}\right)$, we define their sum as $Z=X+Y$, where $\underline{Z}^{\alpha}:=\underline{X}^{\alpha}+\underline{Y}^{\alpha}$ and $\bar{Z}^{\alpha}:=\bar{X}^{\alpha}+\bar{Y}^{\alpha}$ for all $\alpha \in[0,1]$.

To any real number $a \in \mathbb{R}^{3}$, we can assign a fuzzy number $a_{1} \in L\left(\mathbb{R}^{3}\right)$, which is defined by:

$$
a_{1}(x)=\left\{\begin{array}{cc}
1, & \text { if } x=a, \\
0, & \text { otherwise }
\end{array}\right\} .
$$


An order interval in $L\left(\mathbb{R}^{3}\right)$ is defined by $[X, Y]:=$ $\left\{Z \in L\left(\mathbb{R}^{3}\right): X \leq Z \leq Y\right\}$, where $X, Y \in L\left(\mathbb{R}^{3}\right)$.

A set $E$ of fuzzy numbers is called convex if $\lambda \mu_{1}+$ $(1-\lambda) \mu_{2} \in E$ for all $\lambda \in[0,1]$ and $\mu_{1}, \mu_{2} \in E$.

\section{Definitions and Preliminaries}

In this section, we present some preliminary definitions and results related to intuitionistic fuzzy metric triple sequence spaces and that will be used throughout the article.

\section{Definition 2.1}

The five-tuple $(X, \mu, v, *, \diamond)$ is said to be an Intuitionistic Fuzzy Metric Space (for short, IFMS) if $X$ is a vector space, $*$ is a continuous $t$-norm, $\diamond$ is a continuous $t$-conorm and $\mu, v$ are fuzzy sets on $X \times(0, \infty)$ satisfying the following conditions for every $x ; y \in x$ and $s, t>0$ :

(1) $\mu(x, t)+v(x, t) \leq 1$,

(2) $\mu(x, t)>0$,

(3) $\mu(x, t)=1$ if and only if $x=0$,

(4) $\mu(\alpha x, t)=\mu\left(x, \frac{t}{|\alpha|}\right)$ for each $\alpha \neq 0$,

(5) $\mu(x, t) * \mu(y, s) \leq \mu(x+y, t+s)$,

(6) $\mu(x, \cdot):(0, \infty) \rightarrow[0,1]$ is continuous,

(7) $\lim _{t \rightarrow \infty} \mu(x, t)=1$ and $\lim _{t \rightarrow 0} \mu(x, t)=0$;

(8) $v(x, t)<1$,

(9) $v(x, t)=0$ and if and only if $x=0$,

(10) $v(\alpha x, t)=v\left(x, \frac{t}{|\alpha|}\right)$ for each $\alpha \neq 0$,

(11) $v(x, t) \diamond v(y, s) \geq v(x+y, t+s)$,

(12) $v(x, \cdot):(0, \infty) \rightarrow[0,1]$ is continuous,

(13) $\lim _{t \rightarrow \infty} v(x, t)=0$ and $\lim _{t \rightarrow 0} v(x, t)=1$.

In this case $(\mu, v)$ is called intuitionistic fuzzy metric.

\section{Definition 2.2}

Let $(X, \mu, v, *, \diamond)$ be an rough intuitionistic fuzzy metric space and $\beta$ be a non negative real number. A triple sequence $x=\left(x_{m} n k\right)$ in $X$ is said to be convergent to $L \in X$ with respect to the intuitionistic fuzzy metric $(\mu, v)$ if, for every $\epsilon \in(0,1)$ and $t>0$, there exists $m_{0} n_{0} k_{0} \in \mathbb{N}$ such that $\mu\left(x_{\text {mnk }}-L, t\right)>1-(\beta+\epsilon)$ and $v\left(x_{m n k}-L, t\right)<\beta+\epsilon$ for all $m \geq m_{0}, n \geq n_{0}, k \geq k_{0}$.

It is denoted by $(\mu, v)-\lim x=\mathrm{L}$.

\section{Definition 2.3}

Let $(X, \mu, v, *, \diamond)$ be an rough intuitionistic fuzzy metric space and $\beta$ be a non negative real number. A triple sequence $x=\left(x_{m n k}\right)$ in $X$ is said to be a Cauchy sequence with respect to the rough intuitionistic fuzzy metric $(\mu, v)$ if, for every $\epsilon \in(0,1)$ and $t>0$, there exists $m_{0} n_{0} k_{0} \in \mathbb{N}$ such that $\mu\left(x_{m n k}-x_{u v w}, t\right)>1-(\beta+\epsilon)$ and $v\left(x_{m n k}-x_{u v w}, t\right)<\beta+\epsilon$ for all $m, n, k, u, v, w \geq m_{0} n_{0} k_{0}$.

\section{Definition 2.4}

Let $(X, \mu, v, *, \diamond)$ be an rough intuitionistic fuzzy metric space. For $t>0$, the open ball $B(x, r, t)$ with center $x \in X$ and radius $r \in(0,1)$ is defined as:

$$
B(x, r, t)=\left\{\begin{array}{l}
y \in X: \mu(x-y, t) \\
>1-\operatorname{rand} v(x-y, t)<r
\end{array}\right\}
$$

\section{Definition 2.5}

If $X$ be a non-empty set. Then a family $I \subset P(X)$ of subsets of $X$ is called an ideal in $X$ if and only if:

(i) $\emptyset \in I$

(ii) $A, B \in I$ implies $A \subset B \in I$

(iii) For each $A \in I$ and $B \subset A$ we have $B \in I$

where, $P(X)$ is the power set of $X$.

\section{Definition 2.6}

If $X$ be a non-empty set. Then a non-empty family of sets $\mathcal{F} \subset P(X)$ is called a filter on $X$ if and only if:

(i) (i) $\emptyset \notin \mathcal{F}$

(ii) $A, B \in \mathcal{F}$ implies that $A \cap B \in \mathcal{F}$

(iii) For each $A \in \mathcal{F}$ and $B \supset A$ we have $B \in \mathcal{F}$

An ideal $I$ is called non-trivial if $I \neq \emptyset$. A non-trivial ideal $I \subset P(X)$ is called an admissible ideal in $X$ if and only if it contains all singletons, i.e., if $I \supset\{\{x\}: x \in X\}$.

\section{Remark 2.1}

For each ideal $I$ there is a filter $\mathcal{F}(I)$ which corresponding to $I$ (filter associate with ideal $I$ ), that is:

$$
\mathcal{F}(I)\left\{K \subseteq X: K^{C} \in I\right\} \text {, where } K^{C}=X \backslash K \text {. }
$$

\section{Definition 2.7}

The triple sequence $\theta_{i, \ell, j}=\left\{\left(m_{i}, n_{\ell}, k_{j}\right)\right\}$ is called triple lacunary if there exist three increasing sequences of integers such that:

$$
m_{0}=0, h_{i}=m_{i}-m_{r-1} \rightarrow \infty \text { as } i \rightarrow \infty
$$


and:

$$
\begin{aligned}
& n_{0}=0, \overline{h_{\ell}}=n_{\ell}-n_{\ell-1} \rightarrow \infty \text { as } \ell \rightarrow \infty . \\
& k_{0}=0, \overline{h_{j}}=k_{j}-k_{j-1} \rightarrow \infty \text { as } j \rightarrow \infty .
\end{aligned}
$$
by:

Let $m_{i, \ell, j}=m_{i} n_{\ell} k_{j}, h_{i, \ell, j}=h_{i} h_{\ell} h_{j}$ and $\theta_{i, \ell_{j}}$ be determined

$$
\begin{aligned}
& I_{i, \ell, j}=\left\{\begin{array}{l}
(m, n, k): m_{i-1}<m<m_{i} \text { and } n_{\ell-1} \\
<n<n_{\ell} \text { and } k_{j-1}<k \leq k_{j}
\end{array}\right\}, \\
& q_{k}=\frac{m_{k}}{m_{k-1}}, \overline{q_{\ell}}=\frac{n_{\ell}}{n_{\ell-1}}, \overline{q_{j}}=\frac{k_{j}}{k_{j-1}}
\end{aligned}
$$

\section{Definition 2.8}

Let $I \subset P(\mathbb{N})$ and let $(X, \mu, v, *, \diamond)$ be an rough intuitionistic fuzzy metric space and $\beta$ be a non negative real number. A triple sequence $\mathrm{x}=\left(x_{m n k}\right)$ in $X$ is said to be Lacunary $I$-convergent to $L \in X$ with respect to the rough intuitionistic fuzzy metric $(\mu, v)$ if, for every $\epsilon \in(0$, 1) and $t>0$, the set:

$$
\begin{aligned}
& \left\{\begin{array}{l}
r \in \mathbb{N}: \frac{1}{h_{r s t}} \sum_{(m n k) \in J_{r}} \mu\left(x_{m n k}-L, t\right) \leq(1-(\beta+\epsilon)) \\
\operatorname{or} \frac{1}{h_{r s t}} \sum_{(m n k) \in J_{r}} v\left(x_{m n k}-L, t\right) \geq \beta+\epsilon
\end{array}\right\} \in I . \\
& Z_{(\mu, v)}^{I_{\theta}}=\left\{x=\left(x_{m n k}\right) \in \omega:\left\{r \in \mathbb{N}: \frac{1}{h_{r s t}} \sum_{(m n k) \in J_{r}} \mu\left(x_{m n k}^{\prime}-L, t\right) \leq 1-(\beta+\epsilon) \operatorname{or} \frac{1}{h_{r s t}} \sum_{(m n k) \in J_{r}} v\left(x_{m n k}^{\prime}-L, t\right) \geq \beta+\epsilon\right\} \in I\right\} \\
& Z_{0(\mu, v)}^{I_{\theta}}=\left\{x=\left(x_{m m k}\right) \in \omega:\left\{r \in \mathbb{N}: \frac{1}{h_{r s t}} \sum_{(m n k) \in J_{r}} \mu\left(x_{m n k}^{\prime}, t\right) \leq 1-(\beta+\epsilon) \text { or } \frac{1}{h_{r s t}} \sum_{(m n k) \in J_{r}} v\left(x_{m n k}^{\prime}, t\right) \geq \beta+\epsilon\right\} \in I\right\}
\end{aligned}
$$

Also, we define an open ball with center $x^{\prime}$ and radius $r$ with respect to $t$ as follows:

$$
B\left(x^{\prime}, r, t\right)=\left\{y=\left(y_{k}\right) \in \omega:\left\{r \in \mathbb{N}: \frac{1}{h_{r}} \sum_{k \in J_{r}} \mu\left(x^{\prime}-y^{\prime}, t\right) \leq 1-(\beta+\epsilon) \operatorname{or} \frac{1}{h_{r}} \sum_{k \in J_{r}} v\left(x^{\prime}-y^{\prime}, t\right) \geq \beta+\epsilon\right\} \in I\right\}
$$

\section{Theorem 3.1}

The space $Z_{(\mu, v)}^{I_{\theta}}$ and $Z_{0(\mu, v)}^{I_{\theta}}$ are linear space.

\section{Proof}

We prove the result for $Z_{0(\mu, v)}^{I_{\theta}}$. The proof for other space will follow Similarly. Let $x^{\prime}=\left(x_{m n k}^{\prime}\right), y^{\prime}=\left(y_{m n k}^{\prime}\right) \in Z_{(\mu, v)}^{I_{\theta}}$ and let $\alpha, \gamma$ be scalars. Then for a given $\epsilon>0$, we have: 


$$
\begin{aligned}
& A_{1}=\left\{m, n, k \in \mathbb{N}: \frac{1}{h_{r s t}} \sum_{(m n k) \in J_{r}} \mu\left(x_{m n k}^{\prime}-L_{1}, \frac{t}{2|\alpha|}\right) \leq 1-(\beta+\epsilon) \text { or } \frac{1}{h_{r s t}} \sum_{(m n k) \in J_{r}} v\left(x_{m n k}^{\prime}-L_{1}, \frac{t}{2|\alpha|}\right) \geq \beta+\epsilon\right\} \\
& A_{2}=\left\{(m, n, k) \in \mathbb{N}: \frac{1}{h_{r s t}} \sum_{(m n k) \in J_{r}} \mu\left(y_{m n k}^{\prime}-L_{2}, \frac{t}{2|\gamma|}\right) \leq 1-\epsilon \text { or } \frac{1}{h_{r s t}} \sum_{(m n k) \in J_{r}} v\left(y_{m n k}^{\prime}-L_{2}, \frac{t}{2|\alpha|}\right) \geq \epsilon\right\} \in I, \\
& A_{1}^{c}=\left\{(m, n, k) \in \mathbb{N}: \frac{1}{h_{r s t}} \sum_{(m n k) \in J_{r}} \mu\left(x_{m n k}^{\prime}-L_{1}, \frac{t}{2|\gamma|}\right)>1-\left(\beta+\epsilon \text { or } \frac{1}{h_{r s t}} \sum_{m n k \in J_{r}} v\left(x_{m n k}^{\prime}-L_{1}, \frac{t}{2|\alpha|}\right)<\beta+\epsilon\right\} \in I\right. \\
& A_{2}^{c}=\left\{(m, n, k) \in \mathbb{N}: \frac{1}{h_{r s t}} \sum_{(m n k) \in J_{r}} \mu\left(y_{m n k}^{\prime}-L_{2}, \frac{t}{2|\gamma|}\right)>1-\epsilon \text { or } \frac{1}{h_{r s t}} \sum_{(m n k) \in J_{r}} v\left(y_{m n k}^{\prime}-L_{2}, \frac{t}{2|\alpha|}\right)<\beta+\epsilon\right\} \in F(I)
\end{aligned}
$$

Define the set $A_{3}=A_{1} \cap A_{2}$, so that $A_{3} \in I$. it follows that $A_{3}^{c}$ is a non-empty set in $\mathcal{F}(I)$. We shall show that for each $\left(x^{\prime}{ }_{m n k}\right),\left(y_{m n k}^{\prime}\right) \in Z^{I_{\theta}}(\mu, v)$.

Let $m \in A_{3}^{I}$. In this case:

$$
\begin{aligned}
& \frac{1}{h_{r s t},(a b c) \in J_{r}} \sum_{J_{r}} \mu\left(x_{a b c}^{\prime}-L_{1} \frac{t}{2|\alpha|}\right)>1-(\beta+\epsilon) \\
& \operatorname{or} \frac{1}{h_{r s t},(a b c) \in J_{r}} \sum_{r} v\left(x_{a b c}^{\prime}-L_{1} \frac{t}{2|\alpha|}\right)<\beta+\epsilon
\end{aligned}
$$

and:

$$
\begin{aligned}
& \frac{1}{h_{r s t},(a b c) \in J_{r}} \sum_{J_{r}} \mu\left(y_{a b c}^{\prime}-L_{2} \frac{t}{2|\gamma|}\right)>1-(\beta+\epsilon) \\
& \operatorname{or} \frac{1}{h_{r s t}} \sum_{(a b c) \in J_{r}} v\left(y_{a b c}^{\prime}-L_{2} \frac{t}{2|\gamma|}\right)<\beta+\epsilon
\end{aligned}
$$

we have:

$$
\begin{aligned}
& \frac{1}{h_{r s t,}} \sum_{(a b c) \in J_{r}} \mu\left(\left(\alpha x_{a b c}^{\prime}+\beta y_{a b c}^{\prime}\right)-\left(\alpha L_{1}+\beta L_{2}\right), t\right) \\
& \geq \frac{1}{h_{r s t}} \sum_{(a b c) \in J_{r}} \mu\left(\alpha x_{a b c}^{\prime}+\alpha L_{1}, \frac{t}{2}\right) * \frac{1}{h_{r s t}} \sum_{a b c \in J_{r}} \mu\left(\gamma y_{a b c}^{\prime}+\beta L_{2}, \frac{t}{2}\right) \\
& =\frac{1}{h_{r s t}} \sum_{(a b c) \in J_{r}} \mu\left(x_{a b c}^{\prime}+L_{1}, \frac{t}{2|\alpha|}\right) * \frac{1}{h_{r s t}} \sum_{(a b c) \in J_{r}} \mu\left(y_{a b c}^{\prime}+L_{2}, \frac{t}{2|\gamma|}\right) \\
& >(1-(\beta+\epsilon)) *(1-(\beta+\epsilon))=(1-(\beta+\epsilon)) .
\end{aligned}
$$

and:

$$
\begin{aligned}
& \frac{1}{h_{r s t}} \sum_{(a b c) \in J_{r}} \mu\left(\left(\alpha x_{a b c}^{\prime}+\beta y_{a b c}^{\prime}\right)-\left(\alpha L_{1}+\gamma L_{2}\right), t\right) \\
& \geq \frac{1}{h_{r s t}} \sum_{(a b c) \in J_{r}} v\left(\alpha x_{a b c}^{\prime}-\alpha L_{1}, \frac{t}{2}\right) \diamond \frac{1}{h_{r s t}} \sum_{(a b c) \in J_{r}} v\left(\gamma y_{m}^{\prime}-\gamma L_{2}, \frac{t}{2}\right) \\
& =\frac{1}{h_{r s t}} \sum_{(a b c) \in J_{r}} v\left(x_{a b c}^{\prime}-L_{1}, \frac{t}{2|\alpha|}\right) \diamond \frac{1}{h_{r s t}} \sum_{(a b c) \in J_{r}} v\left(y_{a b c}^{\prime}-L_{2}, \frac{t}{2|\gamma|}\right) \\
& >\beta+\epsilon \diamond \beta+\epsilon=\beta+\epsilon .
\end{aligned}
$$

this is implies that:

$$
A_{3}^{c} \subset\left\{\begin{array}{l}
\left.(m n k) \in \mathbb{N}: \frac{1}{h_{r s t}} \sum_{(m n k) \in J_{r}} \mu\left(\alpha x_{m n k}^{\prime}+\gamma y_{m n k}^{\prime}\right)-\left(\alpha L_{1}+\gamma L_{2}\right)\right) \\
>1-(\beta+\epsilon) \\
o r \frac{1}{h_{r s t}} \sum_{\left((m n k) \in J_{r}\right.} v\left(x_{m n k}^{\prime}+\gamma y_{m n k}^{\prime}\right)-\left(\alpha L_{1}+\gamma L_{2}\right)<\beta+\epsilon
\end{array}\right\}
$$

Hence $Z_{(\mu, v)}^{I_{\theta}}$ is a linear space.

\section{Theorem 3.2}

Every open ball $B\left(x^{\prime}, t, \beta\right)$ is open in $Z_{(\mu, v)}^{I_{\theta}}$.

\section{Proof}

Let $B\left(x^{\prime}, t, \beta\right)$ be an open ball with center $x^{\prime}$ and radius $\beta$ with respect to $t$. That is:

$$
\begin{aligned}
& B\left(x^{\prime}, t, \beta\right) \\
& =\left\{\begin{array}{l}
(m, n, k) \in \mathbb{N}: \frac{1}{h_{r s t}} \sum_{(m, n, k) \in J_{r}} \mu\left(x_{m n k}^{\prime}-L, t\right) \leq 1-\beta \\
o r \frac{1}{h_{r s t}} \sum_{(m, n, k) \in J_{r}} v\left(x_{m n k}^{\prime}-L, t\right) \geq \beta
\end{array}\right\} \in I .
\end{aligned}
$$

Let $y^{\prime} \in B^{c}\left(x^{\prime}, t, \beta\right)$. Then:

$$
\frac{1}{h_{r s t}} \sum_{(m, n, k) \in J_{r}} \mu\left(x^{\prime}-y^{\prime}, t\right)>1-\beta \text { and } \frac{1}{h_{r s t}} \sum_{(m, n, k) \in J_{r}} v\left(x^{\prime}-y^{\prime}, t\right)<\beta
$$

Since $\mu\left(x^{\prime}-y^{\prime}, t\right)>1-\beta$, there exists $t_{0} \in(0,1)$ such that:

$$
\begin{aligned}
& \mu\left(x^{\prime}-y^{\prime}, t_{0}\right)>1-\beta \\
& \text { and } \frac{1}{h_{r s t}} \sum_{(m, n, k) \in J_{r}} \frac{1}{h_{r s t}} \sum_{(m, n, k) \in J_{r}} v\left(x^{\prime}-y^{\prime}, t_{0}\right)<\beta
\end{aligned}
$$

Putting $\beta_{0}=\frac{1}{h_{r s t}} \sum_{(m, n, k) \in J_{r}} \mu\left(x^{\prime}-y^{\prime}, t_{0}\right)$. We have $\beta_{0}>$ $1-\beta$, there exists $s \in(0,1)$ such that: 


$$
\beta_{0}>1-s>1-\beta .
$$

For $\beta_{0}>1-s$, we have $\beta_{1}, \beta_{2} \in(0,1)$ such that:

$$
\beta_{0} * \beta_{1}>1-s \text { and }\left(1-\beta_{0}\right) \diamond\left(1-\beta_{2}\right) \leq s .
$$

Putting $\beta_{3}=\max \left\{\beta_{1}, \beta_{2}\right\}$, consider the ball $B^{c}\left(y^{\prime}, 1-\right.$ $\left.\beta_{3}, t-t_{0}\right)$. We prove that:

$$
B^{c}\left(y^{\prime}, 1-r_{3}, t-t_{0}\right) \subset B^{c}\left(x^{\prime}, r, t\right) .
$$

Let $z^{\prime} \in B^{c}\left(y^{\prime}, 1-\beta_{3}, t-t_{0}\right)$ :

$$
\begin{aligned}
& \frac{1}{h_{r s t}} \sum_{(m, n, k) \in J_{r}} \mu\left(y^{\prime}-z^{\prime}, t-t_{0}\right)>\beta_{3} \\
& \text { and } \frac{1}{h_{r s t}} \sum_{(m, n, k) \in J_{r}} v\left(y^{\prime}-z^{\prime}, t-t_{0}\right)<\beta_{3} .
\end{aligned}
$$

Therefore:

$$
\begin{aligned}
& \frac{1}{h_{r s t}} \sum_{(m, n, k) \in J_{r}} \mu\left(x^{\prime}-z^{\prime}, t\right) \geq \frac{1}{h_{r s t}} \sum_{(m, n, k) \in J_{r}} \mu\left(x^{\prime}-y^{\prime}, t_{0}\right) \\
& * \frac{1}{h_{r s t}} \sum_{(m, n, k) \in J_{r}} \mu\left(y^{\prime}-z^{\prime}, t-t_{0}\right) \\
& \geq\left(\beta_{0} * \beta_{3}\right) \geq\left(\beta_{0} * \beta_{1}\right) \geq(1-s)>(1-\beta)
\end{aligned}
$$

and:

$$
\begin{aligned}
& \frac{1}{h_{r s t}} \sum_{(m, n, k) \in J_{r}} v\left(x^{\prime}-z^{\prime}, t\right) \\
& \leq \frac{1}{h_{r s t}} \sum_{(m, n, k) \in J_{r}} v\left(x^{\prime}-y^{\prime}, t_{0}\right) \diamond \frac{1}{h_{r s t}} \sum_{(m, n, k) \in J_{r}} v\left(y^{\prime}-z^{\prime}, t-t_{0}\right) \\
& \leq\left(1-\beta_{1}\right) \diamond\left(1-\beta_{3}\right) \leq\left(1-\beta_{0}>\left(1-\beta_{2}\right)<s<\beta\right.
\end{aligned}
$$

Thus $z^{\prime} \in B^{c}\left(x^{\prime}, t, \beta\right)$ and hence $B^{c}\left(y^{\prime}, 1-\beta_{3}, t-t_{0} \subset\right.$ $\left.B^{c}\left(x^{\prime}, t, \beta\right)\right)$.

\section{Remark 3.1.}

$Z_{(\mu, v)}^{I_{\theta}}$ is RIFMS.

Define $\tau_{(\mu, v)}=\left\{A \subset Z_{(\mu, v)}^{I_{o}}\right.$ for each $x \in A, \exists t>0$ and $\beta \in(0,1)$ s.t $\left.B^{c}\left(x^{\prime}, t, \beta\right) \subset A\right\}$.

Then $\tau_{(\mu, v)}$ is topology on $Z_{(\mu, v)}^{I_{\theta}}$.

\section{Theorem 3.3}

The topology $\tau_{(\mu, v)}$ on $Z_{(\mu, v)}^{I_{\theta}}$ is first countable.

\section{Proof}

$\left\{B\left(x^{\prime}, \frac{1}{n^{3}}, \frac{1}{n^{3}}\right): n=1,2,3, \ldots\right\}$ is a local base at $x^{\prime}$ the topology $\tau_{(\mu, v)}$ is first countable.

\section{Theorem 3.4.}

$Z_{(\mu, v)}^{I_{\theta}}$ and $Z_{0(\mu, \nu)}^{I_{\theta}}$ are Hausdroff spaces.

\section{Proof}

We prove the result for $Z_{(\mu, v)}^{I_{\theta}}$. Similarly the result can be proved for $Z_{0(\mu, v)}^{I_{\theta}}$. Let $x^{\prime}, y^{\prime} \in Z_{(\mu, \nu)}^{I_{\theta}}$ such that $x^{\prime} \neq y^{\prime}$. Then:

$0<\frac{1}{h_{r s t}} \sum_{(m, n, k) \in J_{r}} \mu\left(x^{\prime}-y^{\prime}, t\right)<1$ and $\frac{1}{h_{r s t}} \sum_{(m, n, k) \in J_{r}} v\left(x^{\prime}-y^{\prime}, t\right)<1$.

Putting $\quad \beta_{1}=\frac{1}{h_{r s t}} \sum_{(m, n, k) \in J_{r}} \mu\left(x^{\prime}-y^{\prime}, \quad t\right)$ and $\beta_{2}=$ $\frac{1}{h_{r s t}} \sum_{(m, n, k) \in J_{r}} v\left(x^{\prime}-y^{\prime}, t\right)$ and $r=\max \left\{\beta_{1}, 1-\beta_{2}\right\}$.

For each $\beta_{0} \in(\beta, 1) \exists \beta_{3}$ and $\beta_{4}$ such that:

$$
\beta_{3} * \beta_{4} \geq \beta_{0} \text { and }\left(1-\beta_{3}\right) \diamond\left(1-\beta_{4}\right) \leq\left(1-\beta_{0}\right)
$$

Putting $\beta_{5}=\max \left\{\beta_{3}, 1-\beta_{4}\right\}$ and consider the open ball $B\left(x^{\prime}, 1-\beta_{5} \frac{t}{2}\right)$ and $B\left(y^{\prime}, 1-\beta_{5} \frac{t}{2}\right)$.

Clearly:

$$
B\left(x^{\prime}, 1-\beta_{5} \frac{t}{2} \cap B\left(y^{\prime}, 1-\beta_{5} \frac{t}{2}=\phi\right) .\right.
$$

For if there exists $z^{\prime} \in B\left(x^{\prime}, 1-\beta_{5} \frac{t}{2} \cap B\left(y^{\prime}, 1-\beta_{5} \frac{t}{2}\right)\right.$. Then and:

$$
\begin{aligned}
& \beta_{1}=\frac{1}{h_{r s t}} \sum_{(m, n, k) \in J_{r}} \mu\left(x^{\prime}-y^{\prime}, t\right) \geq \frac{1}{h_{r s t}} \sum_{(m, n, k) \in J_{r}} \mu\left(x^{\prime}-z^{\prime}, \frac{t}{2}\right) \\
& * \frac{1}{h_{r s t}} \sum_{(m, n, k) \in J_{r}} \mu\left(z^{\prime}-y^{\prime}, \frac{t}{2}\right) \\
& \geq \beta_{5} * \beta_{5} \geq \beta_{3} * \beta_{3} \geq \beta_{0}>\beta_{1} .
\end{aligned}
$$

and:

$$
\begin{aligned}
& \beta_{2}=\frac{1}{h_{r s t}} \sum_{(m, n, k) \in J_{r}} v\left(x^{\prime}-y^{\prime}, t\right) \\
& \leq \frac{1}{h_{r s t}} \sum_{(m, n, k) \in J_{r}} v\left(x^{\prime}-z^{\prime}, \frac{t}{2}\right) \diamond \frac{1}{h_{r s t}} \sum_{(m, n, k) \in J_{r}} v\left(z^{\prime}-y^{\prime}, \frac{t}{2}\right) \\
& \leq\left(1-\beta_{5}\right) \diamond\left(1-\beta_{5}\right) \leq\left(1-\beta_{4}\right) \diamond\left(1-\beta_{4}\right) \\
& \leq\left(1-\beta_{0}\right)<\beta_{0}>\beta,
\end{aligned}
$$

which is contradiction. Hence $Z_{(\mu, v)}^{I_{\theta}}$ is Hausdroff space. 


\section{Conclusion}

The triple sequence spaces of rough intuitionistic fuzzy zweier lacunary ideal convergence of sequence have been defined here and some algebraic and topological properties of these spaces are investigated. These definitions and results provide new tools to deal with the convergence problems of triple sequences in the fuzzy settings, occurring in many branches of science and engineering.

\section{Authors Contributions}

All authors of the manuscript have read and agreed to its content and are accountable for all aspects of the accuracy and integrity of the manuscript.

\section{Competing Interests}

The authors declare that they have no competing interests.

\section{References}

Aytar, S., 2008a. Rough statistical convergence. Numer. Funct. Anal. Optimiz., 29: 291-303. DOI: $10.1080 / 01630560802001064$

Aytar, S., 2008b. The rough limit set and the core of a real sequence. Numer. Funct. Anal. Optimiz., 29: 283-290. 10.1080/01630560802001056

Debnath, S., B. Sarma and B.C. Das, 2015. Some generalized triple sequence spaces of real numbers. J. Nonlinear Anal. Optimiz., 6: 71-79.

Dündar, E. and C. Cakan, 2014. Rough I-convergence. Demonstratio Math., 47: 638-651. DOI: $10.2478 /$ dema-2014-0051

Dutta, A.J., A. Esi and B.C. Tripathy, 2013. Statistically convergent triple sequence spaces defined by Orlicz function. J. Math. Anal., 4: 16-22.
Esi, A. and E. Savas, 2015. On lacunary statistically convergent triple sequences in probabilistic normed space. Applied Math. Inform. Sci., 9: 2529-2534. DOI: $10.12785 / \mathrm{amis} / 090537$

Esi, A. and M. Necdet Catalbas, 2014. Almost convergence of triple sequences. Global J. Math. Anal., 2: 6-10. DOI: 10.14419/gjma.v2i1.1709

Esi, A., 2014. On some triple almost lacunary sequence spaces defined by Orlicz functions. Res. Rev.: Discrete Math. Structures, 1: 16-25.

Esi, A., N. Subramanian and A. Esi, 2018. Wijsman rough $I$-convergence limit point of triple sequences defined by a metric function. Annals Fuzzy Math. Inform., 15: 47-57.

Esi, A., S. Araci and M. Acikgoz, 2016. Statistical convergence of bernstein operators. Applied Math. Inform. Sci., 10: 2083-2086. DOI: $10.18576 /$ amis/100610

Phu, H.X., 2001. Rough convergence in normed linear spaces. Numer. Funct. Anal. Optimiz., 22: 199-222. DOI: $10.1081 /$ NFA-100103794

Phu, H.X., 2002. Rough continuity of linear operators. Numer. Funct. Anal. Optimiz., 23: 139-146. DOI: $10.1081 /$ NFA-120003675

Phu, H.X., 2003. Rough convergence in infinite dimensional normed spaces. Numer. Funct. Anal. Optimiz., 24: 285-301. DOI: 10.1081/NFA-120022923

Sahiner, A. and B.C. Tripathy, 2008. Some I related properties of triple sequences. Selcuk J. Applied Math., 9: 9-18.

Sahiner, A., M. Gurdal and F.K. Duden, 2007. Triple sequences and their statistical convergence. Selcuk J. Applied Math., 8: 49-55.

Subramanian, N. and A. Esi, 2015. The generalized tripled difference of $\chi^{3}$ sequence spaces. Global J. Math. Anal., 3: 54-60.

DOI: $10.14419 /$ gjma.v3i2.4412 